\title{
Liver Imaging Techniques: Recognition of Uveal Melanoma Metastases
}

\author{
Claudine Bellerive $^{a} \quad$ Etienne Ouellet $^{b} \quad$ Aya Kamaya $^{b} \quad$ Arun D. Singh $^{a}$

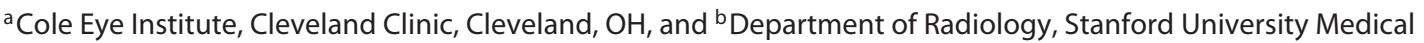 \\ Center, Stanford, CA, USA
}

\section{Keywords}

Liver imaging · Hepatic cyst · Hepatic hemangioma ·

Metastasis · Uveal melanoma

\begin{abstract}
Background: The liver is the most common site for metastases of several primary malignancies including uveal melanoma. Methods: Review of imaging characteristics of incidental common benign liver lesions including hepatic cyst, hemangioma, focal nodular hyperplasia, and hepatic adenoma and contrasting them with uveal melanoma metastases. Results: Benign hepatic lesions may be cystic or, if solid, relatively stable in size over time. For hepatic lesions larger than $10 \mathrm{~mm}$ in size, characteristic imaging features typically allow for confident diagnosis. When lesions are small (less than 10 $\mathrm{mm}$ ), definitive characterization can be difficult. Moreover, lesions smaller than $10 \mathrm{~mm}$ can be difficult to biopsy under ultrasound or computed tomography (CT) guidance, and short-term follow-up will often be useful to assess for stability or progression. Overall, magnetic resonance imaging is more specific than CT scan and at least as sensitive as CT for detecting uveal melanoma liver metastases. Conclusions: New multiple enhancing solid liver lesions should raise suspicion of uveal melanoma liver metastases. Discussion of
\end{abstract}

challenging cases with the radiologist may be beneficial, as pertinent information such as size, location, and molecular prognostication status of the primary tumor can guide radiological interpretation of hepatic lesions.

(c) 2018 S. Karger AG, Basel

\section{Introduction}

The liver is the most common site for metastases in a number of primary malignancies, including colorectal, breast and lung carcinomas [1]. In primary uveal melanoma, up to $95 \%$ of metastases involve the liver $[2,3]$. Numerous noninvasive liver imaging methods are important for staging and systemic surveillance of metastatic disease in patients with uveal melanoma. These modalities include ultrasound (US), contrast-enhanced computed tomography (CT), contrast-enhanced magnetic resonance imaging (MRI), and positron emission tomography (PET), each with its advantages and limitations (Table 1).

Incidental findings are frequent on liver imaging with any of the modalities listed above. Common benign liver lesions include hepatic cyst, hemangioma, focal nodular hyperplasia, and hepatic adenoma (Table 2). According to

\section{KARGER}

(c) 2018 S. Karger AG, Basel

E-Mail karger@karger.com

www.karger.com/oop
Arun D. Singh, MD

Department of Ophthalmic Oncology

Cole Eye Institute, Cleveland Clinic

2022 E 105th St, Cleveland, OH 44106 (USA)

E-Mail singha@ccf.org 
Table 1. Modalities for liver imaging

\begin{tabular}{lll}
\hline Modality & Advantages & Limitations \\
\hline Ultrasound & Noninvasive & Operator dependent \\
& $\begin{array}{l}\text { Wide availability } \\
\text { Inexpensive } \\
\text { Contrast-enhanced (microbubble) } \\
\text { No side effects }\end{array}$ & $\begin{array}{l}\text { Limited by large body habitus } \\
\text { Low spatial resolution }\end{array}$ \\
& Good accessibility & \\
\hline CT scan & Rapid & Low contrast resolution \\
& Reproducible & Ionizing radiation \\
& Whole-body imaging & Requires iodinated intravenous \\
& High contrast resolution & Requires absence of renal insufficiency \\
\hline MRI & Provides more specific characterization & Claustrophobia \\
& No ionizing radiation & Metallic implants are a contraindication \\
& & Requires absence of renal insufficiency \\
& Expensive and time consuming \\
\hline PET scan & Whole-body imaging & Low resolution \\
& & Higher radiation exposure \\
& & Nonspecific appearance of the lesions \\
& & Expensive
\end{tabular}

In general, ultrasonography cannot detect lesions less than $10 \mathrm{~mm}$. CT and MRI have comparable spatial resolution but MRI offers superior contrast resolution.

Table 2. Frequent incidental findings on liver ultrasonographic imaging

\begin{tabular}{lcl}
\hline Entity & Frequency, \% & Appearance on ultrasonography \\
\hline Hepatic cyst & $0.06-17.8$ & Anechoic lesion (multiple) \\
Hepatic hemangioma & $0.1-20$ & Homogenously hyperechoic lesion (single) \\
Focal nodular hyperplasia & $0.2-3.2$ & Nondiagnostic appearancea \\
Hepatic adenoma & $0.04-1.5$ & Nondiagnostic appearancea \\
\hline
\end{tabular}

${ }^{\text {a }}$ Requires contrast-enhanced CT or MRI for a definitive diagnosis.

published clinical and pathological studies, the prevalence of hepatic cyst and hemangioma in the general population ranges from 0.06 to $17.8 \%$ and from 0.1 to $20 \%$, respectively [4-7]. Focal nodular hyperplasia (FNH) and hepatic adenoma are less frequent $(0.2-3.2 \%$ and $0.04-1.5 \%$, respectively) [8-10]. Autopsy series have reported a high prevalence of benign focal liver lesions of $52 \%$ in patients with no known history of a malignant tumor [11].

In the setting of primary uveal melanoma, differentiating common benign liver lesions from true metastatic lesions is critical. Incidental liver lesions detected on routine imaging may lead to an unnecessary aggressive workup, such as percutaneous liver biopsy with a potential morbidity rate of $0.13-6.4 \%$ (for indeterminate lesions) [12]. Although biopsy may be needed in select cases for a definitive diagnosis, many benign liver lesions as well as uveal melanoma metastases have highly specific imaging and enhancement characteristics which can allow confident diagnosis with imaging alone. The purpose of this paper is to review characteristic imaging features of common liver lesions with emphasis on differentiation from uveal melanoma metastases.

\section{Methods}

Imaging Protocols

The specifics of an imaging protocol may vary due to several factors such as available equipment, patient weight, and allergy to contrast. For abdominal US, the selection of the US probes (curved, 2.5-6 MHz; phased, 2.5-5 MHz) is based on the body habitus. Con- 


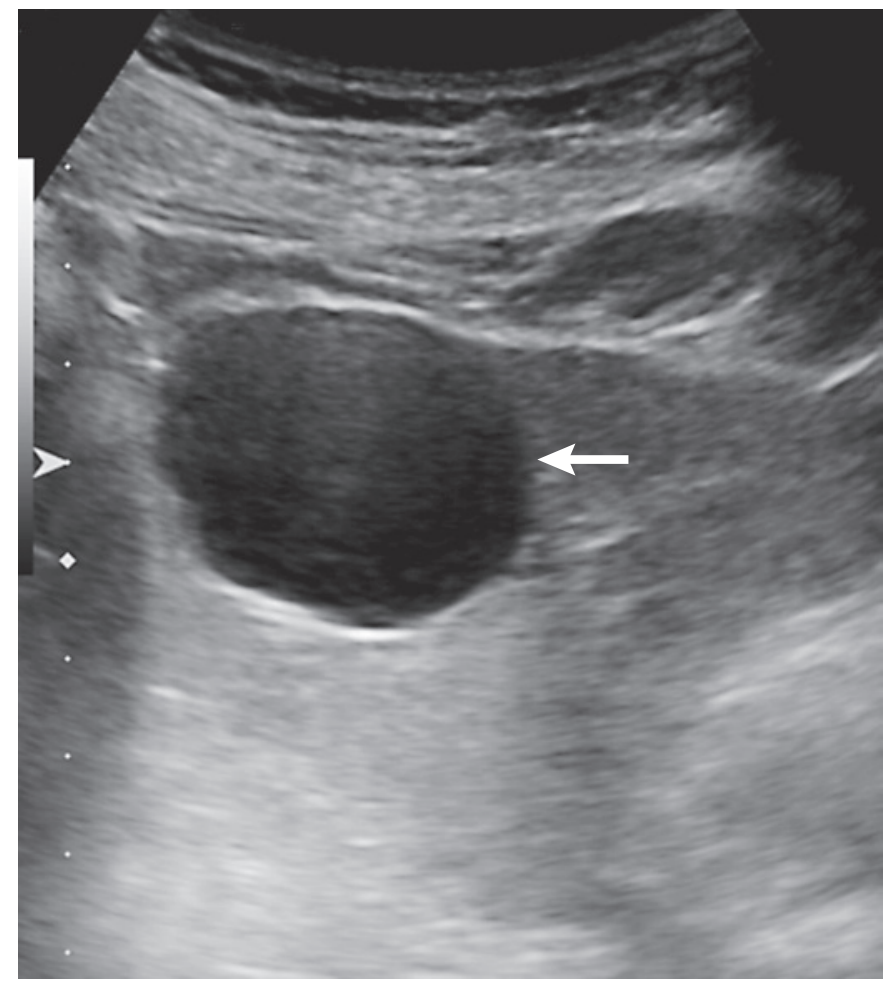

Fig. 1. Typical liver cyst on ultrasound. The lesion is anechoic, with a well-defined posterior wall and posterior acoustic enhancement (arrow).

trast-enhanced CT scan of the abdomen and pelvis is performed using a multidetector CT, scanning from just above the dome of the diaphragm to the symphysis pubis. The patients receive iodinated intravenous contrast, based on weight, 30-60 s before image acquisition including an arterial phase, a portal venous phase (70 s), and a delayed phase (3-5 min). Images are typically acquired with 0.625 mm collimation with multiplanar reformations. MRI images of the abdomen are acquired on 1.5- or 3-T MRI. Multiplanar and multisequence (T2-weighted, T1-weighted, diffusion-weighted imaging, and postcontrast) imaging are performed. Approximately $60 \mathrm{~min}$ following the intravenous administration of fludeoxyglucose-18 $(7.2 \mathrm{mCi}), \mathrm{PET}$ and noncontrast CT images are acquired from the skull base to the proximal thigh using a combined PET-CT scanner. A low-dose CT scan is performed for attenuation correction and anatomic correlation only. Images are reconstructed and reviewed in the axial, coronal, and sagittal planes.

\section{Imaging Features}

Benign Liver Lesions

The hepatic cyst is a developmental lesion that does not directly communicate with the biliary tree [13]. On US, hepatic cysts appear as well-defined anechoic lesions (often multiple) with associated posterior acoustic enhancement and absent internal vascularity (Fig. 1) [13, 14]. On CT, a hepatic cyst can be characterized confidently when it appears as a nonenhancing hypodense lesion with Hounsfield units measuring $<20$ (corresponding to fluid density) [13]. On MRI, hepatic cysts typically demonstrate uniform

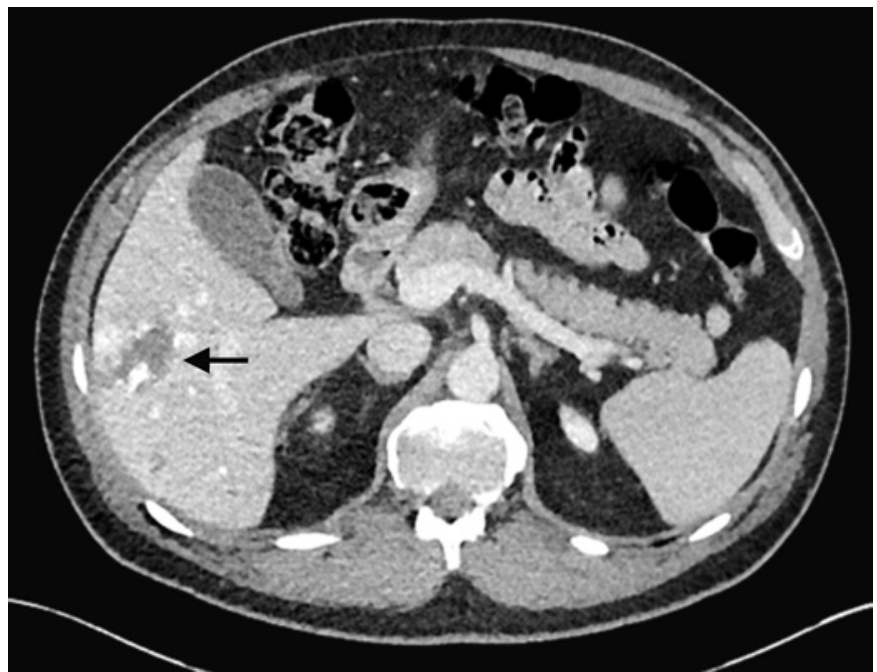

Fig. 2. Solitary hepatic lesion with peripheral discontiguous nodular enhancement on CT compatible with a hemangioma (arrow). This is an incidental finding in a 48 -year-old male.

high T2 signal intensity and do not enhance on T1-weighted images after intravenous administration of gadolinium chelate contrast. US, CT or MRI alone is sufficient to make an accurate diagnosis of a simple cyst.

Hepatic hemangioma, also known as cavernous hemangioma, is the most common benign solid hepatic tumor [9]. Hepatic hemangiomas, usually solitary, are well-circumscribed lesions containing blood-filled spaces lined by endothelium on a thin fibrous stroma [15]. Occasionally, giant hemangiomas $(>4-6 \mathrm{~cm})$ may cause nonspecific abdominal symptoms related to mass effect [16]. In two-thirds of the cases, hemangiomas appear as homogenously hyperechoic masses on US [17]. In some cases, and especially when larger in size, hemangiomas may have a hypoechoic or mixed echogenicity appearance with a thin hyperechoic rim. This imaging appearance, although less common, is still considered characteristic for hemangioma, leading to its description as the "typical atypical hemangioma" appearance [15]. On contrast-enhanced US, CT or MRI, hemangiomas may demonstrate either a characteristic pattern of peripheral nodular discontinuous enhancement that progresses centripetally to uniform enhancement, or immediate uniform enhancement which follows the blood pool on subsequent phases, termed "flash-filling hemangioma" (Fig. 2) [16]. On T2-weighted MRI, hemangiomas tend to have a markedly hyperintense appearance, also called "light bulb bright" appearance. This is a distinguishing feature from metastases, which tend to be mildly hyperintense on T2-weighted images (similar in signal intensity to the spleen).

Hepatic adenoma is a relatively rare primary hepatic tumor that occurs more frequently in women on oral contraceptives, in men who are treated with exogenous steroids, or in patients with glycogen storage disease. Hepatic adenoma is a benign liver neoplasm that has a $5-10 \%$ risk of malignant transformation into hepatocellular carcinoma (HCC) [18]. Three distinct histologic subtypes have been identified: inflammatory hepatocellular adenomas, hepatocyte nuclear factor $1 \alpha$-mutated hepatocellular adenomas, and 


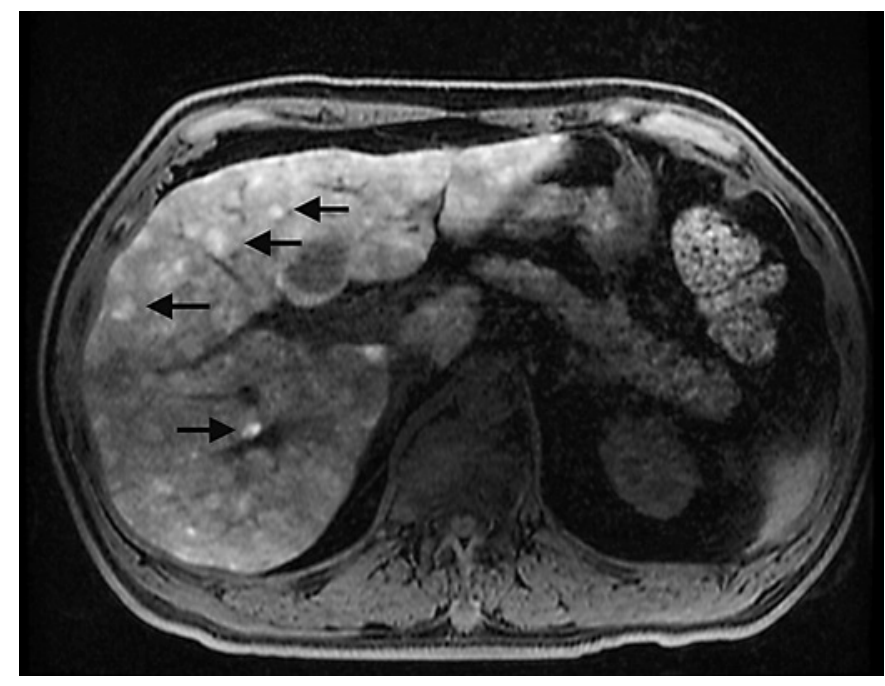

Fig. 3. Numerous T1-weighted fat suppressed hyperintense lesions on MRI compatible with melanoma metastases (arrows).

$\beta$-catenin-mutated hepatocellular adenomas [18]. On US, hepatic adenomas typically appear solid and may be isoechoic, hypoechoic, or hyperechoic (depending on lipid content) in appearance. CT/ MRI diagnostic features of hepatic adenoma depend on the subtype with a combination of fat, hemorrhage, and arterial enhancement [19].

FNH consists of nonneoplastic hepatocytes in disordered arrays surrounding a central scar with anomalous vessels [20]. On routine US, FNH often appears isoechoic compared to background liver. [19]. On contrast-enhanced US, FNH has a characteristic spoke wheel vascularity with a feeding vessel enhancing from the center to the periphery. Early arterial enhancement that becomes isoenhancing to background liver parenchyma on portal venous phase and delayed phases is typical of FNH on CT imaging. Some FNHs contain a central scar, which may demonstrate delayed enhancement and bright signal on T2-weighted MRI. Often FNH is barely perceptible on cross-sectional imaging, especially when arterialphase imaging is not included in the acquisition, leading to its monicker, the "stealth lesion." Definitive diagnosis of an FNH can be made on gadoxetate-enhanced MRI in which the lesion retains contrast to an equal or greater extent compared to background liver due to the presence of hepatocytes and lack of biliary excretion [21].

\section{Malignant Liver Lesions}

HCC represents the most frequent primary hepatic malignancy [22]. Eighty percent of HCCs develop in patients with a cirrhotic liver. Other risk factors for HCC include chronic hepatitis B virus infection and aflatoxin exposure. On US, HCC is typically solid and hypoechoic in appearance although a minority may appear isoechoic or hyperechoic compared to background liver. A solid lesion $\geq 10$ $\mathrm{mm}$ in size in a cirrhotic liver requires definitive characterization with a contrast-enhanced multiphasic study. On contrast-enhanced US, HCC enhances early with late (after $60 \mathrm{~s}$ ) and weak washout. On multiphasic CT or MRI, a typical HCC demonstrates arterial-phase hyperenhancement and washout (hypoenhance-
Table 3. Differential diagnosis of $\mathrm{T} 1$ hyperintense liver lesions

\begin{tabular}{ll}
\hline $\begin{array}{l}\text { Fat or lipid- } \\
\text { containing }\end{array}$ & Nonfat-containing \\
\hline
\end{tabular}

Hepatocellular

carcinoma Hematoma/hemorrhagic metastases

Adenoma

Lipoma

Melanoma metastases

Angiomyolipoma

Adapted from Furlan et al. [42].

a The lesions will be hypointense on a fat suppressed T1weighted image. Please note that not all HCCs and adenomas contain fat or lipid.

ment compared to background liver) on delayed-phase images. In addition, HCC may have a pseudocapsule and occasionally may contain intratumoral fat [22]. HCC may invade the portal veins or hepatic veins, termed "tumor in vein," which is more suggestive of a hepatic primary malignancy than metastatic disease [23]. The LI-RADS (Liver Imaging Reporting and Data System) aids the radiologist in assessing lesions in a cirrhotic liver or other risk factors for HCC (for example, certain populations with chronic hepatitis $B$ virus) and contains imaging criteria for contrast-enhanced US and $\mathrm{CT}$, and MRI that, if met, can allow for the definitive diagnosis of HCC that can preclude the need for confirmatory biopsy [24].

Liver metastasis occurs 18 times more frequently than primary malignant liver neoplasm [16]. Multiple solid (i.e., noncystic) lesions in the liver suggest a metastatic process, as only $20 \%$ of liver metastases present as a solitary lesion [17]. On unenhanced US, metastases may have variable echogenicity depending on the primary tumor. On contrast-enhanced US, metastases tend to enhance very early, briefly with either a rim or diffuse pattern followed by rapid washout, leading to a "punched out" washout appearance by $60 \mathrm{~s}$ after the start of intravenous contrast [25]. On contrast-enhanced CT and MRI, metastases can be hypovascular or hypervascular depending on the primary tumor. The arterial phase of contrast-enhanced cross-sectional imaging is critical in detecting hypervascular metastases from uveal melanoma, sarcoma, thyroid carcinoma, or choriosarcoma.

Liver metastasis from uveal melanoma tends to be multiple and demonstrates avid enhancement due to its hypervascular nature. In addition, melanoma metastasis has a characteristic intrinsic hyperintense T1 signal on precontrast images (due to melanin), which helps to differentiate melanoma metastases from other hypervascular metastatic lesions (Fig. 3) [16, 20, 26]. Melanoma metastases to other parts of the body (for example, nodal metastases) tend to have similar radiologic features. Other liver lesions can also be hyperintense on precontrast T1-weighted images (Table 3) [11]. When hepatic lesions are intrinsically $\mathrm{T} 1$ hyperintense on precontrast images (such as uveal melanoma metastases), it can be difficult to determine if there is postcontrast enhancement. For this reason, it is important to obtain subtracted images, obtained by removing the signal of the precontrast images from the postcontrast images (eliminating background signal) to detect contrastinduced enhancement [27]. 
Table 4. General imaging features that should raise suspicion of uveal melanoma metastases

Solid lesion

Enhancing lesion

Multiple lesions

Absent on prior imaging studies

Enlargement of lesion when compared to prior imaging studies

Primary tumor with high risk of metastasis (ciliary body

location, large size, adverse molecular profile)

\section{Uveal Melanoma}

Systemic Staging

Less than $4 \%$ of patients with uveal melanoma have occult liver metastases at the time of diagnosis [28]. Metastases are predictive of a poor outcome once diagnosed, with a reported median survival time of approximately 6 months $[3,29]$. In addition, patients with uveal melanoma are at an increased risk of developing second primary malignant tumors that could be detected during initial staging or subsequent surveillance [30]. Thus, initial accurate staging is crucial for determining the appropriate treatment strategies for the patient. The characterization of small (less than $5 \mathrm{~mm}$ ) and focal liver lesions is often difficult and their presence does not necessarily correspond to metastases.

\section{Systemic Surveillance}

There is no consensus regarding the surveillance approach of asymptomatic hepatic metastases in patients with uveal melanoma. Even though liver function tests have been proven irrelevant in the diagnosis of hepatic metastases from uveal melanoma [31], wide variation exists concerning the choice of the imaging examination and the frequency of the surveillance [32].

In general, the sensitivity of detecting small lesions decreases as lesions are smaller, with lesions less than $5 \mathrm{~mm}$ in size most challenging with all modalities (US, CT, and MRI). CT typically has better spatial resolution than MRI but MRI offers superior contrast resolution [32,33]. Marshall et al. [34] reported hepatic metastases at a presymptomatic stage in $92 \%$ of 90 high-risk patients with uveal melanoma who underwent 6-monthly MRI. However, previous research has also indicated that liver US offers a high specificity for detection of metastases $[35,36]$. Choudhary et al. [26] showed that the negative predictive value of the initial US abnormal findings when confirmed by CT or MRI increased to $99 \%$. US features of large (more than $10 \mathrm{~mm}$ ), multiple solid lesions, and new onset or enlarging lesions should raise suspicion of metastases and should be characterized by contrast-enhanced CT or MRI (Table 4).

\section{Discussion}

Benign liver lesions are frequently seen in patients with preexisting malignancy. Jones et al. [37] have investigated the significance of small hepatic lesions $(15 \mathrm{~mm}$ or less) detected by contrast-enhanced abdominal CT scans in 1,454 consecutives outpatients. The lesions were identified in 254 patients. Even though $82 \%$ of the patients had known extrahepatic malignant tumor, $51 \%$ of the lesions were classified as benign on the basis of further imaging, the stability of the lesions over time or, in rare cases, the result of a percutaneous biopsy. Similarly, a retrospective review of 2,978 CT scans has reported high prevalence $(80 \%)$ of benign small hepatic lesions in patients with prior history of extrahepatic malignant tumor with only $11.6 \%$ of the lesions identified as metastases [38]. The incidental findings of the Committee of American College of Radiologists provide an algorithm for the management of liver incidental lesions based on their size and the patient's level of risk (Fig. 4) [39]. Patients who have a history of primary malignant tumor are considered as high-risk individuals.

In general, imaging features of a benign liver lesion include stability, cystic appearance, absence of enhancement, diffuse hyperintensity on T2-weighted, and retention of the contrast agent gadoxetic acid on late hepatobiliary phase images (15-20 $\mathrm{min}$ ) for FNH. The presence of multiple solid lesions should raise suspicion of metastases. When hepatic lesions are larger than $10 \mathrm{~mm}$ in size, imaging features typically allow for confident diagnosis. When lesions are small (less than $5 \mathrm{~mm}$ ), definitive characterization can be difficult. Overall, MRI is more specific than CT scan and similar in sensitivity to CT for detecting liver metastases, particularly, when the lesions are smaller than $10 \mathrm{~mm}[40,41]$. Comparison with prior imaging studies to assess for recent onset or enlargement is often essential in establishing the diagnosis. Moreover, lesions smaller than $10 \mathrm{~mm}$ can be difficult to biopsy under US or CT guidance, and short-term follow-up will often be useful to assess for stability or growth. Discussing challenging cases with the radiologist may also be beneficial, as pertinent clinical history such as size, location, and molecular prognostication status of the primary tumor can guide radiological interpretation of hepatic lesions.

\section{Statement of Ethics}

The study complied with the guidelines for human studies and animal welfare regulations. The subject gave informed consent and the study protocol was approved by the institute's committee on human research.

\section{Disclosure Statement}

The authors have no relevant conflicts of interest to declare related to this work. 


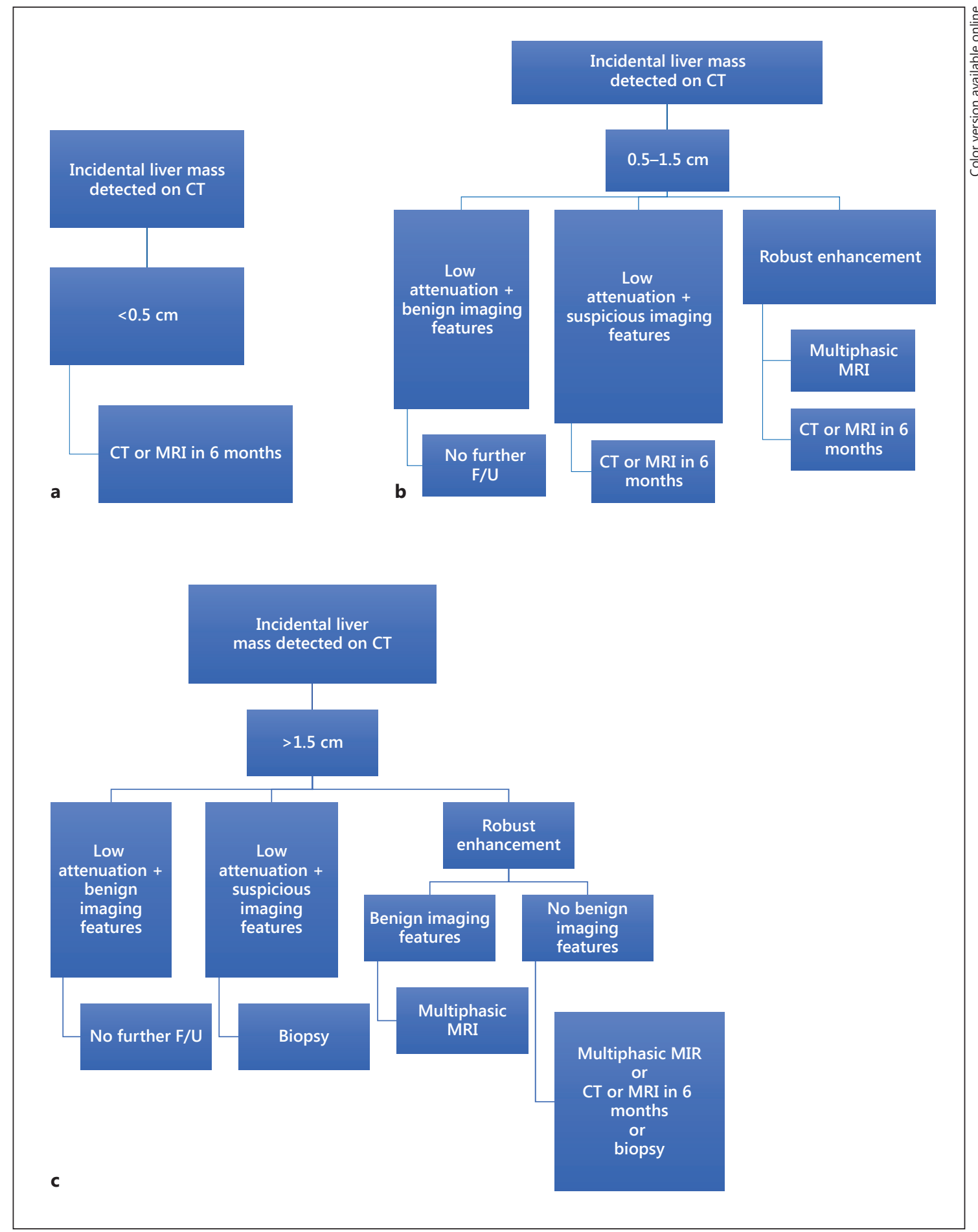

Fig. 4. Adapted algorithm for managing incidental liver mass findings being less than $0.5 \mathrm{~cm} \mathrm{(a),} \mathrm{between} 0.5$ and $1.5 \mathrm{~cm}$ (b) and more than $1.5 \mathrm{~cm}$ (c) in high-risk patients [39]. F/U, follow-up. 


\section{References}

1 Abbruzzese J, Abbruzzese M, Lenzi R: Analysis of a diagnostic strategy for patients with suspected tumors of unknown origin. J Clin Oncol 1995;13:2094-2103.

2 Diener-West M, Reynolds S, Agugliaro D: Development of metastatic disease after enrolment in the COMS trials for treatment of choroidal melanoma: Collaborative Ocular Melanoma Study Group Report No 26. Arch Ophthalmol 2005;123:1639-1643.

3 Gragoudas ES, Egan K, Seddon J, Glynn R, Walsh S, Finn S: Survival of patients with metastases from uveal melanoma. Ophthalmology 1991;98:383-390.

4 Rungsinaporn K, Phaisakamas T: Frequency of abnormalities detected by upper abdominal ultrasound. J Med Assoc Thai 2008;91: 1072-1075.

5 Kaltenbach T, Engler P, Kratzer W, et al: Prevalence of benign focal liver lesions: ultrasound investigation of 45,319 hospital patients. Abdom Radiol (NY) 2016;41:25-32.

6 Gaines P, Sampson M: The prevalence and characterization of simple hepatic cysts by ultrasound examination. Br J Radiol 1989;62: 335-337.

7 Massironi S, Branchi F, Rossi R, et al: Hepatic hemangioma in celiac patients: data from a large consecutive series. Gastroenterol Res Pract 2015;2015:749235

8 Buscarini E, Danesino C, Plauchu H, et al: High prevalence of hepatic focal nodular hyperplasia in subjects with hereditary hemorrhagic telangiectasia. Ultrasound Med Biol 2004;30:1089-1097.

9 Karhumen PJ: Benign hepatic tumors and tumor-like conditions in men. J Clin Pathol 1986;39:183-188.

10 Marin D, Galluzzo A, Plessier A, Brancatelli G, Valla D, Vilgrain V: Focal nodular hyperplasia-like lesions in patients with cavernous transformation of the poral vein: prevalence, MR findings and natural history. Eur Radiol 2011;21:2074-2082.

11 Washington K: Masses of the liver; in Odze R, Goldblum JR (eds): Surgical Pathology of the GI Tract, Liver, Biliary Tract and Pancreas, ed 2. New York, Elsevier, 2009.

12 Thampanitchawong P, Piratvisuth T: Liver biopsy: complications and risk factors. World J Gastroenterol 1999;5:301-304.

13 Mortelé K, Ros P: Cystic focal liver lesions in the adult: differential CT and MR imaging features. Radiographics 2001;21:895-910.

14 Lantinga M, Gevers T, Drenth J: Evaluation of hepatic cystic lesions. World J Gastroenterol 2013;19:3543-3554.

15 Vilgrain V, Boulos L, Vullierme MP, Denys A, Terris B, Menu Y: Imaging of atypical hemangiomas of the liver with pathologic correlation. Radiographics 2000;20:379-397.
16 Silva A, Evans J, McCullough A, Jatoi M, Vargas $\mathrm{H}$, Hara A: MR imaging of hypervascular liver masses: a review of current techniques. Radiographics 2009;29:385-402.

17 Assy N, Nasser G, Djibre A, Beniashvili Z, Elias S, Zidan J: Characteristics of common solid liver lesions and recommendations for diagnostic workup. World J Gastroenterol 2009;15:3217-3227.

18 Katabathina V, Menias C, Shanbhogue A, Jagirdar J, Paspulati R, Prasad S: Genetics and imaging of hepatocellular adenomas: 2011 update. Radiographics 2011;31:1529-1543.

19 Mohajer K, Frydrychowicz A, Robbins JB, Loeffler AG, Reed TD, Reeder SB: Characterization of hepatic adenoma and focal nodular hyperplasia with gadoxetic acid. J Magn Reson Imaging 2012;36:686-696.

20 Fowler K, Brown J, Narra V: Magnetic resonance imaging of focal liver lesions: approach to imaging diagnosis. Hepatology 2011;54: 2227-2237.

21 Shamsi K, De Schepper A, Degryse H, Deckers F: Focal nodular hyperplasia of the liver: radiologic findings. Abdom Imaging 1993;18: 32-38.

22 Choi J, Lee J, Sirlin C: CT and MR imaging diagnosis and staging of hepatocellular carcinoma: part I. Development, growth, and spread: key pathologic and imaging aspects. Radiology 2014;272:635-654.

23 Choi J, Lee J, Sirlin C: CT and MR imaging diagnosis and staging of hepatocellular carcinoma: part II. Extracellular agents, hepatobiliary agents, and ancillary imaging features. Radiology 2014;273:30-50.

24 Mitchell D, Bruix J, Sherman M, Sirlin C: LI-RADS (Liver Imaging Reporting and Data System): summary, discussion, and consensus of the LI-RADS Management Working Group and future directions. Hepatology 2015;61:1056-1065.

25 Jang HJ, Kim TK, Wilson SR: Imaging of malignant liver masses: characterization and detection. Ultrasound Q 2006;22:19-29.

26 Choudhary M, Gupta A, Bena J, Emch T, Singh A: Hepatic ultrasonography for surveillance in patients with uveal melanoma. JAMA Ophthalmol 2016;134:174-180.

27 Mainardi LT, Passera KM, Lucesoli A, Potepan P, Setti E, Musumeci R: A method for dynamic subtraction MR imaging of the liver. BMC Med Imaging 2006;6:5.

28 Finger P, Kurli M, Reddy S, Tena L, Pavlick A: Whole body PET/CT for initial staging of choroidal melanoma. Br J Ophthalmol 2005; 89:1270-1274.
29 Kath R, Hayungs J, Bornfeld N, Sauerwein W, Hoffken K, Seeber S: Prognosis and treatment of disseminated uveal melanoma. Cancer 1993;72:2219-2223.

30 Lains I, Bartosch C, Mondim V, et al: Second primary neoplasms in patients with uveal melanoma: a SEER database analysis. Am J Ophthalmol 2016;165:54-64.

31 Mouriaux F, Diorio C, Bergeron D, Berchi C, Rousseau A: Liver function testing is not helpful for early diagnosis of metastatic uveal melanoma. Ophthalmology 2012;119:15901595.

32 Francis J, Patel S, Gombos D, Carvajal R: Surveillance options for patients with uveal melanoma following definitive management. Am Soc Clin Oncol Educ Book 2013:382-387.

33 Lin E, Alessio A: What are the basic concepts of temporal, contrast, and spatial resolution in cardiac CT? J Cardiovasc Comput Tomogr 2009;3:403-408.

34 Marshall E, Romaniuk C, Ghaneh P: MRI in the detection of hepatic metastases from highrisk uveal melanoma: a prospective study in 188 patients. Br J Ophthalmol 2013;97:159163

35 Eskelin S, Pyrhonen S, Summanen P: Screening for metastatic malignant melanoma of the uvea revisited. Cancer 1999;85:1151-1159.

36 Diener-West M, Reynolds S, Agugliaro D, et al: Screening for metastasis from choroidal melanoma: the Collaborative Ocular Melanoma Study Group Report 23. J Clin Oncol 2004; 22:2438-2444.

37 Jones E, Chezmar J, Nelson R, Bernardino M: The frequency and significance of small (less than or equal to $15 \mathrm{~mm}$ ) hepatic lesions detected by CT. AJR Am J Roentgenol 1992;158: 535-539.

38 Schwartz L, Gandras E, Colangelo S, Ercolani M, Panicek D: Prevalence and importance of small hepatic lesions found at CT in patients with cancer. Radiology 1999;210:71-74.

39 Berland L, Silverman S, Gore R: Managing incidental findings on abdominal CT: white paper of the ACR incidental findings committee. J Am Coll Radiol 2010;7:754-773.

40 Semelka RC, Martin DR, Balci C, Lance T: Focal liver lesions: comparison of dual-phase CT and multisequence multiplanar MR imaging including dynamic gadolinium enhancement. J Magn Reson Imaging 2001;13:397401.

41 Balasubramanya R, Selvarajan SK, Cox M, et al: Imaging of ocular melanoma metastasis. $\mathrm{Br}$ J Radiol 2016;89:20160092.

42 Furlan A, Marin D, Bae KT, Lagalla R, Agnello F, Bazzocchi M, Brancatelli G: Focal liver lesions hyperintense on T1-weighted magnetic resonance images. Semin Ultrasound CT MR 2009;30:436-449. 